\title{
Structural and Chemical Basis for Anticancer Activity of a Series of $\beta$-Tubulin Ligands: Molecular Modeling and 3D QSAR Studies
}

\author{
Livia B. Salum, ${ }^{a}$ Luiz C. Dias ${ }^{b}$ and Adriano D. Andricopulo $*, a$ \\ ${ }^{a}$ Laboratório de Química Medicinal e Computacional, Centro de Biotecnologia Molecular Estrutural, \\ Instituto de Física de São Carlos, Universidade de São Paulo, 13560-970 São Carlos-SP, Brazil \\ ${ }^{b}$ Instituto de Química, Universidade Estadual de Campinas, CP 6154, 13083-970 Campinas-SP, Brazil
}

\begin{abstract}
Uma estratégia importante para a terapia do câncer é o planejamento de modulares que interferem na dinâmica dos microtúbulos através de sua ligação específica à subunidade $\beta$ da tubulina. No presente trabalho, estudos de análise comparativa dos campos moleculares (CoMFA) foram realizados com uma série de análogos do discodermolídeo com ação antimitótica. Resultados significativos foram obtidos $\left(\mathrm{CoMFA}_{(\mathrm{i})}, q^{2}=0,68, r^{2}=0,94\right.$ CoMFA $_{(\mathrm{ii})}, q^{2}=0,63, r^{2}=0,91$ ), indicando a elevada consistência interna e externa dos modelos gerados empregando duas estratégias independentes de alinhamento estrutural. Os modelos foram validados externamente com um conjunto teste e os valores preditos apresentaram boa concordância com os resultados experimentais. Os modelos de QSAR e os mapas de contorno 3D forneceram importantes informações sobre as bases químicas e estruturais envolvidas no processo de reconhecimento molecular dessa família de análogos do discodermolídeo, sendo uma valiosa ferramenta no planejamento de novos moduladores específicos da $\beta$-tubulina com potente atividade antitumoral.
\end{abstract}

An important approach to cancer therapy is the design of small molecule modulators that interfere with microtubule dynamics through their specific binding to the $\beta$-subunit of tubulin. In the present work, comparative molecular field analysis (CoMFA) studies were conducted on a series of discodermolide analogs with antimitotic properties. Significant correlation coefficients were obtained $\left(\mathrm{CoMFA}_{(\mathrm{i})}, q^{2}=0.68, r^{2}=0.94\right.$; $\left.\mathrm{CoMFA}_{(\mathrm{ii})}, q^{2}=0.63, r^{2}=0.91\right)$, indicating the good internal and external consistency of the models generated using two independent structural alignment strategies. The models were externally validated employing a test set, and the predicted values were in good agreement with the experimental results. The final QSAR models and the 3D contour maps provided important insights into the chemical and structural basis involved in the molecular recognition process of this family of discodermolide analogs, and should be useful for the design of new specific $\beta$-tubulin modulators with potent anticancer activity.

Keywords: cancer, drug design, discodermolide, microtubule, $\beta$-tubulin

\section{Introduction}

A rational approach to cancer therapy involves the design of small molecule ligands that interfere with microtubule dynamics through their specific binding to the $\beta$-subunit of tubulin, leading to mitotic arrest and cell death. ${ }^{1-6}$ Taxol (paclitaxel, Figure 1), a highly functionalized diterpenoid isolated from Taxus brevifolia (Pacific Yew tree), was the first compound recognized to interact specifically and reversibly with the $\beta$-subunit of the tubulin heterodimer, promoting microtubule stabilization and consequently, blocking cells

*e-mail: aandrico@if.sc.usp.br in the mitotic phase of the cell cycle. The unique mechanism of action as a microtubule-stabilizing antimitotic agent (MSAA) is responsible by the extraordinary clinical success achieved by Taxol and related taxanes in the treatment of a variety of cancers. Although taxanes are the most prominent among the known MSAAs, their scarceness, poor pharmacokinetic properties, high systemic toxicity and resistance have led to the identification of novel compounds having similar mechanisms of action. ${ }^{6-10}$ These include non-taxane microtubule-stabilizing natural products, such as discodermolide, epothilone and dictyostatin (Figure 1). These promising anticancer agents competitively inhibit the binding of paclitaxel to tubulin polymers, indicating 
an overlapping binding site in the $\beta$-tubulin cavity, which can accommodate a variety of structurally diverse MSAAs in unique and independent ways. ${ }^{11-15}$

The marine polyketide discodermolide is one of the most potent MSAAs known. In addition to its potent antiproliferative and apoptosis-inducing activities, discodermolide is more water soluble than paclitaxel and retains substantial activity against taxane-resistant cell lines. These physicochemical and biological characteristics have qualified discodermolide as a lead compound for the development of new, more effective and safer anticancer agents. ${ }^{13-16}$
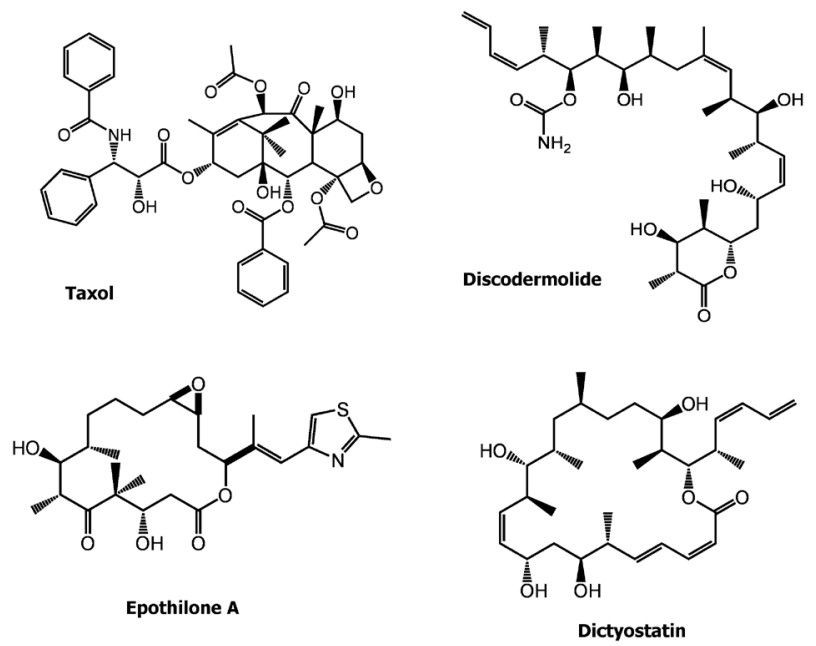

Figure 1. Natural products as microtubule-stabilizing antimitotic agents.

An important strategy in the design of new $\beta$-specific tubulin modulators is to identify key properties of the chemical structure related to their ability to induce a cytotoxic response as a consequence of modulation of microtubule functions through tubulin-binding. Structure and ligand-based drug design approaches have become fundamental components of modern drug discovery. ${ }^{17,18}$ Quantitative structure-activity relationship (QSAR) methods have been successfully employed to assist the design of new small molecule drug candidates, ranging from enzyme inhibitors to receptor ligands. ${ }^{19-26}$ However, there is only a small number of QSAR studies found in the literature for taxoid-site tubulin modulators and, to the best of our knowledge, no 3D QSAR investigation of discodermolide analogs has been reported to date. ${ }^{25,27-30}$ This proves the importance of QSAR studies involving this class of tubulin modulators.

As part of our ongoing research program aimed at designing new $\beta$-tubulin modulators, a rational structurebased design strategy was employed to investigate the molecular recognition patterns required for specific $\beta$-tubulin binding, as schematically shown in Figure 2. In the present study, three-dimensional QSAR comparative molecular field analysis (3D QSAR CoMFA) ${ }^{31}$ models were developed for a series of synthetic discodermolide analogs using two different structural alignment methods in order to explore the high conformational flexibility of the compounds. In the CoMFA method, it is possible to represent the relationships between molecular properties (steric and electrostatic) and biological activity in the 3D protein environment of the $\beta$-tubulin binding site, indicating potential regions for obtaining specificity. The final 3D QSAR models along with the information gathered from 3D contour maps provided important insights into the structural and chemical basis for potent microtubule stabilization and antitumoral properties of this series of discodermolide derivatives. The identification of key intermolecular features associated with affinity and specificity should be a valuable tool for the design of promising candidates for clinical development.

\section{Experimental}

\section{Data set}

The data set used for the QSAR analyses contains 42 discodermolide analogs. ${ }^{32-37}$ The structures and corresponding $\mathrm{pIC}_{50}$ values $\left(-\log \mathrm{IC}_{50}\right.$, where $\mathrm{IC}_{50}$ is the concentration of compound required for $50 \%$ inhibition of A549 lung carcinoma cell growth) for the whole set of ligands are presented in Table 1.

\section{Computational approach}

The QSAR modeling analyses, calculations, and visualizations for CoMFA were performed using the SYBYL 8.0 package (Tripos Inc., St. Louis, MO, USA) running on Red Hat Enterprise Linux workstations. The 3D structures of the small molecule discodermolide modulators were constructed using standard geometric parameters of the SYBYL 8.0 package. Each single optimized conformation of each molecule in the data set was energetically minimized employing the Tripos force field and Gasteiger-Huckel charges. ${ }^{38,39}$ A hierarchical cluster analysis of the data set was carried out with Tsar 3D (Accelrys, San Diego, CA).

\section{Molecular alignment}

Alignment is a crucial component in 3D QSAR studies, and a variety of useful approaches have been described in the literature. ${ }^{18-20}$ Two independent molecular alignment procedures were used in the present study: 


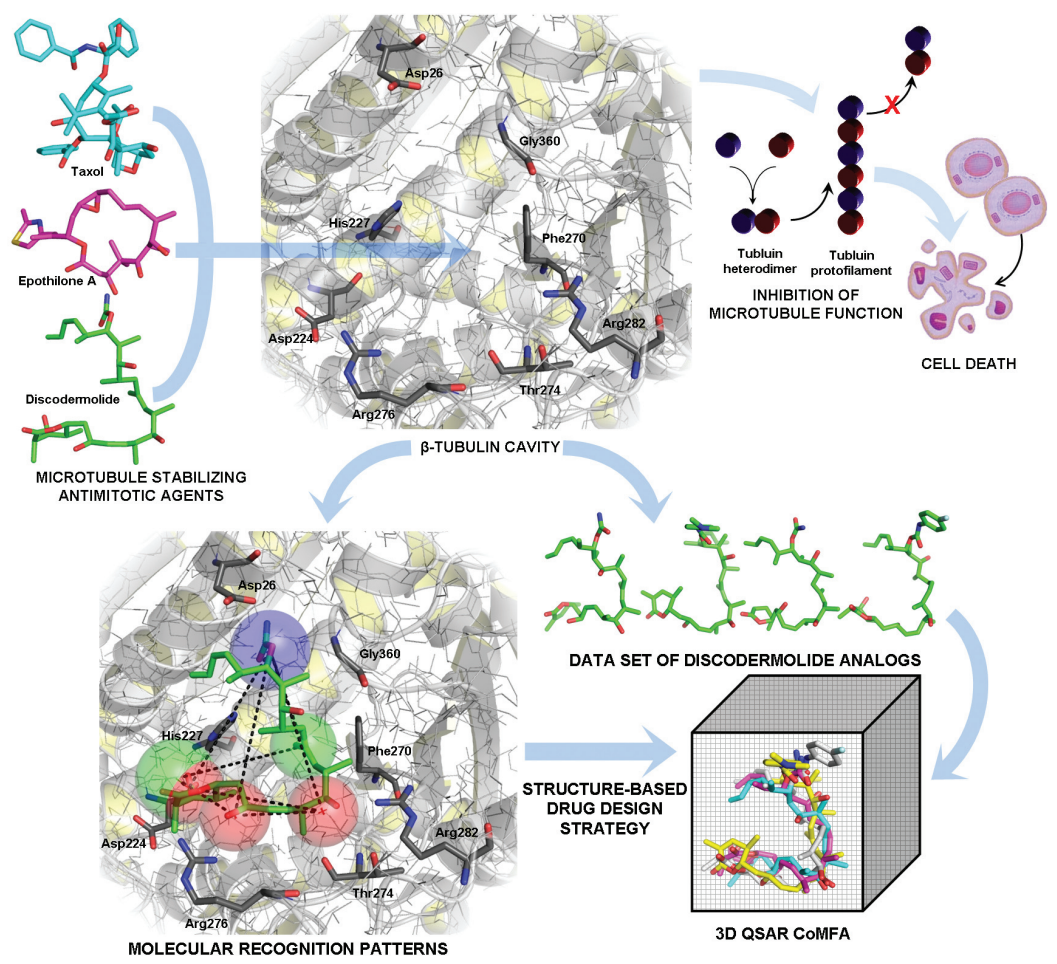

Figure 2. Structure-based design strategy used to investigate the required molecular recognition patterns for specific $\beta$-tubulin binding and anticancer activity.

(i) Rigid-body fit. The lowest energy conformer of discodermolide (compound 25, Table 1) was used as the template structure for the molecular alignment of the series of ligands. All molecules in their respective lowest energy conformations were individually superimposed on the template molecule by using an atom/centroid root mean square (RMS) fitting procedure, the rigid-body fit option in SYBYL 8.0. The alignment and maximal common substructure used for superposition are illustrated in Figure 3.

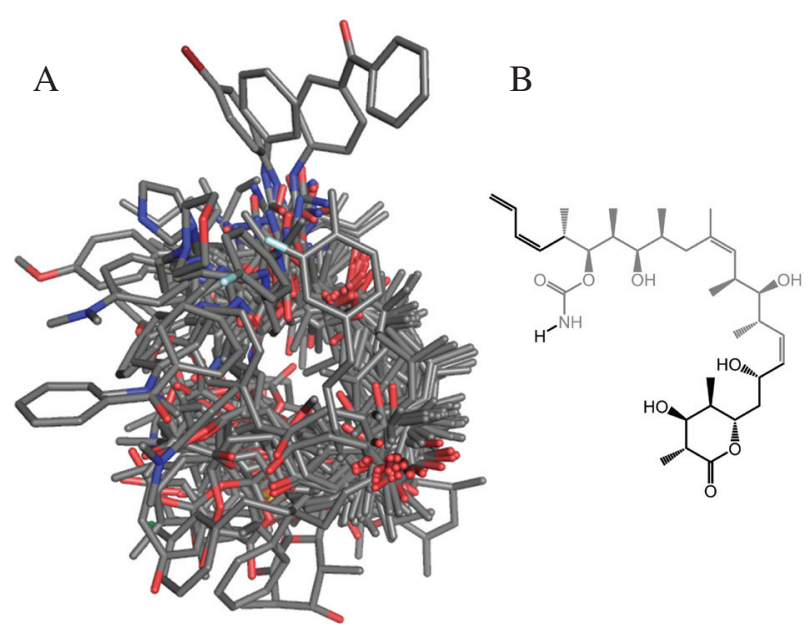

Figure 3. Three-dimensional data set alignment for minimized discodermolide analogs. (A) Rigid-body fit alignment. (B) Template molecule (discodermolide, maximal common substructure shown in gray). (ii) Receptor-based. Predicted binding modes of discodermolide and its analogs into the $\beta$-tubulin cavity have been previously proposed by using an integration of experimental evidences and computer-aided studies. ${ }^{25}$ Docking and scoring protocols as implemented in GOLD 3.1 (Cambridge Crystallographic Data Centre, Cambridge, UK) were used to investigate the possible binding conformations of the ligands within the discodermolide binding domain of $\beta$-tubulin. ${ }^{25,40}$ The X-ray crystallographic data for $\alpha \beta$-tubulin complexed with paclitaxel and epothilone (PDB IDs, 1JFF and 1TVK, respectively) used in the docking studies were retrieved from the Protein Data Bank (PDB). ${ }^{39,40}$ For the calculations, the ligands were removed and hydrogen atoms were added using the biopolymer module as implemented in SYBYL 8.0. The histidine, glutamine, and asparagine residues in the $\beta$-tubulin binding site were manually checked for possible flipped orientation, protonation, and tautomeric states with Pymol (DeLano Scientific, San Carlos, CA, USA). The binding site was centered on the $\mathrm{O} 5$ atom of the taxol and the $\mathrm{O} 2$ atom of epothilone and a radius sphere of $12 \AA$ was considered for the docking procedures, which were repeated 10 times for each molecule due to the stochastic nature of the search algorithm. Default parameters and GOLDscore function were employed in all runs, and only the top-ranked conformations of each compound were considered for the CoMFA studies. The aligned data set is depicted in Figure 4. 
Table 1. Data set of discodermolide analogs employed in the 3D QSAR studies

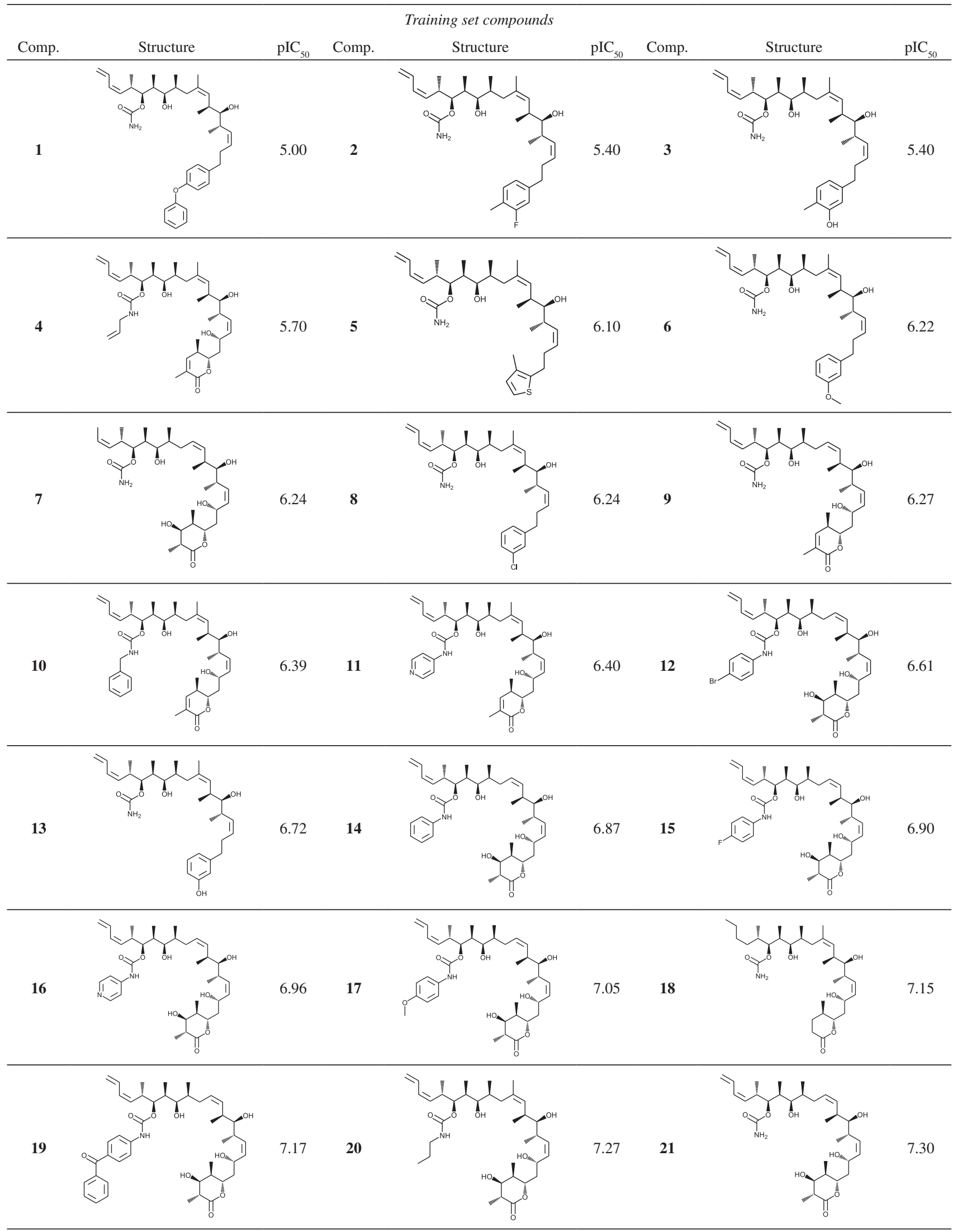


Table 1. Continuation

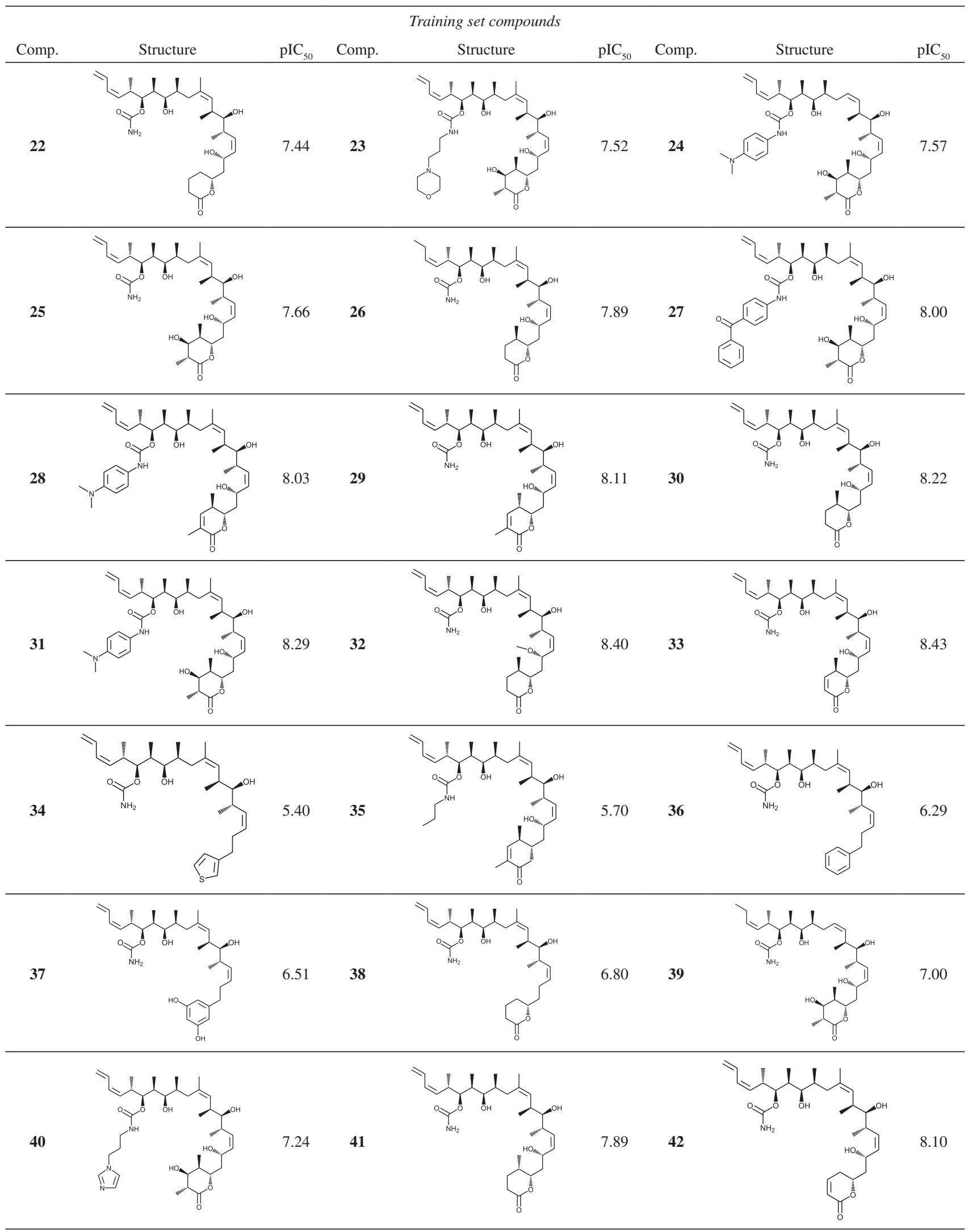




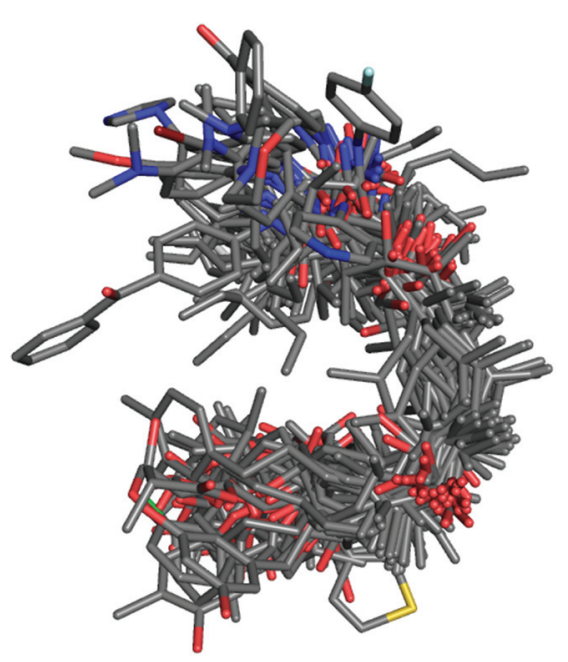

Figure 4. Three-dimensional data set alignment for conformations generated by GOLD 3.1.

\section{D QSAR studies}

To better understand and explore the electrostatic and steric contributions to the binding of the discodermolide analogs, and to build predictive 3D QSAR models, CoMFA studies were performed based on the two molecular alignments described (Figures 3 and 4). Steric and electrostatic properties were calculated according to Lennard-Jones and Coulomb potentials, respectively. The aligned training set molecules were placed in a 3D grid box such that the entire set was included in it. CoMFA steric and electrostatic fields were generated at each grid point with the Tripos force field using a sp ${ }^{3}$ carbon atom probe carrying a +1 net charge. The CoMFA grid spacing of $2.0 \AA$ in the $x, y$, and $z$ directions, and a grid region were automatically generated by the CoMFA routine to encompass all molecules with an extension of $4.0 \AA$ in each direction. ${ }^{38}$ The CoMFA region focusing method was applied to increase the resolution of CoMFA models. The default value of $30 \mathrm{kcal} \mathrm{mol}^{-1}$ was set as the maximum steric and electrostatic energy cutoff. CoMFA descriptors were used as independent variables whereas $\mathrm{pIC}_{50}$ values were used as dependent variables in the partial least squares (PLS) regression analyses to derive the 3D QSAR models. All models were investigated using full crossvalidated $\left(q^{2}\right)$ leave-one-out (LOO) and leave-many-out (LMO) methods, with CoMFA standard options for scaling of variables. The progressive scrambling method was applied to determine the sensitivity of the QSAR models to chance correlations. The models were externally validated with a test set of compounds, which were not considered for QSAR model generation. After generation of the PLS training set models, the dependent variables $\left(\mathrm{pIC}_{50}\right)$ were predicted for the test set compounds, allowing predictive $r^{2}$ values to be determined for individual 3D QSAR models. ${ }^{43,44}$

\section{Results and Discussion}

\section{Chemical and biological data}

3D QSAR CoMFA models were derived for a series of 42 discodermolide analogs for which $\mathrm{IC}_{50}$ values for the inhibition of A549 cell growth were collected (Table 1). The in vitro $\mathrm{IC}_{50}$ values employed in this work were measured under the same experimental conditions, a fundamental requirement for QSAR studies. ${ }^{32-37}$ The $\mathrm{IC}_{50}$ values vary from 3.7 to $10000 \mathrm{nM}$, a factor of about 2700. The discodermolide analogs of the data set present structural variations at the $\mathrm{C}_{21}$-side chain, $\mathrm{C}_{19}$-carbamate group, $\mathrm{C}_{14}$-and $\mathrm{C}_{7}$-substituents, and in the lactone ring, as depicted in Figure 5. The values of $\mathrm{IC}_{50}$ were converted into the corresponding $\mathrm{pIC}_{50}$ values (Table 1), which span approximately three and a half orders of magnitude, and are acceptably distributed across the range of $\mathrm{pIC}_{50}$ values (Figure 6).

The generation of consistent statistical models depends on the quality of both training and test sets in terms of

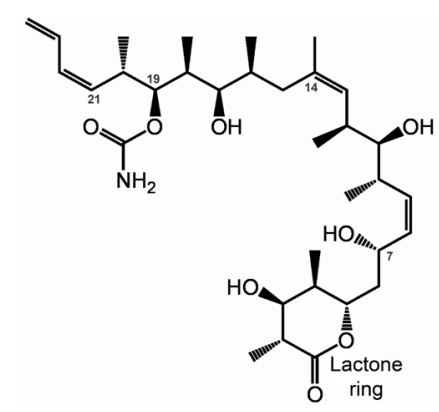

Figure 5. Structural variations presented by the 42 discodermolide analogs of the data set.

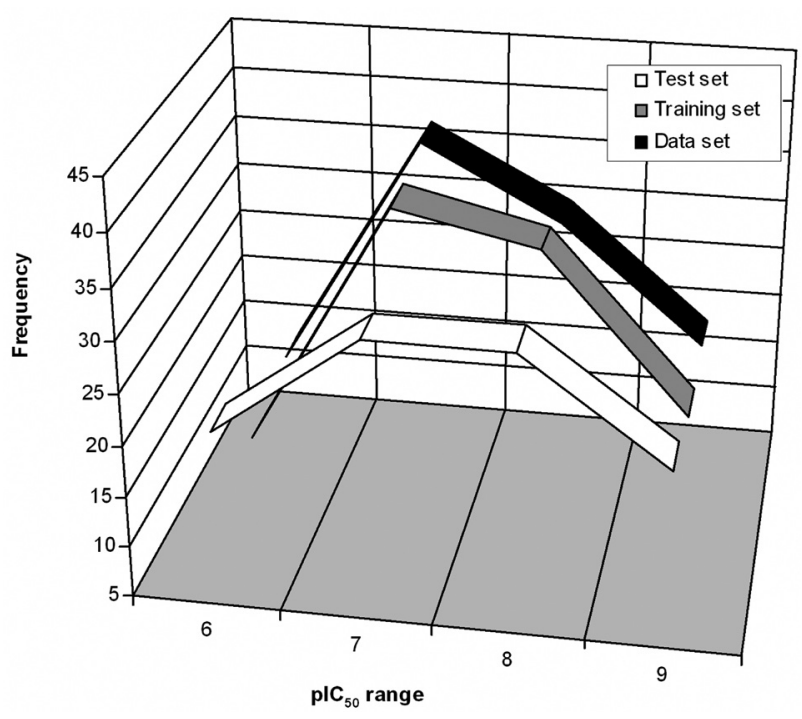

Figure 6. Distribution of $\mathrm{pIC}_{50}$ values for the training set, test set and complete data set. 
structural diversity and property value distributions. From the original data set, 33 compounds (1-33, Table 1) were selected as members of the training set for model generation, whereas the other 9 compounds (34-42, Table 1) were selected as members of the test set for external model validation. The compounds of the training and test sets were carefully selected in order to ensure appropriate property coverage on the entire range of $\mathrm{pIC}_{50}$ values (Figure 6). A statistical cluster analysis confirmed that the composition of both training and test sets is representative of the whole data set, as can be seen in Figure 6. Thus, the data set is appropriate for the purpose of QSAR model development. The same training set was employed for all 3D QSAR analyses, and the predictive ability of the models was assessed by their $q^{2}$ values and external test set predictions (predictive $r^{2}$ values).

\section{Molecular alignment strategies}

The determination of the spatial structural alignments of the discodermolide analogs in the binding pocket of $\beta$-tubulin can critically affect the outcome of the CoMFA studies, since the analyses are highly dependent on the quality of the alignments. 3D structural information on the interaction of small molecule modulators with the $\beta$-tubulin cavity is limited to crystal structures of the complexes of $\alpha \beta$-tubulin dimers with paclitaxel, docetaxel and epothilone A (PBD IDs JFF, 1TUB and 1TVK, respectively). ${ }^{41,42}$ Although tubulindiscodermolide complexes are not available to date, the crystal structures of the known receptor-ligand complexes have provided an important basis for the understanding of the fundamental chemical and structural requirements for $\beta$-tubulin binding by the discodermolide analogs. However, it should be noted that the scenario is more complex due to the high flexibility of the discodermolide system, creating new challenges in the drug design arena. For this reason, two molecular alignment approaches were used in the present study. In the first case we have applied a receptorindependent strategy derived from a rigid superimposition of the minimum energy conformations of the discodermolide analogs (Figure 3), while in the second case we have employed a receptor-based approach (Figure 4).

Taxanes and epothilones have been found to overlap in their occupation of a rather expansive common binding cavity of $\beta$-tubulin, exploiting the binding pocket in a unique and different manner. Considering the experimental evidence that indicates that the $\beta$-tubulin cavity can accommodate discodermolide and its synthetic analogs, as well as a variety of structurally diverse tubulin modulators, the crystal structures of $\beta$-tubulin complexed with paclitaxel (PDB ID 1JFF) and epothilone A (PDB ID 1TVK) were used in the receptor-based molecular docking studies, in such a way to explore the significant conformational flexibility of both receptor cavity and ligands. ${ }^{41,42}$ The two structures were superposed on one another using the $\mathrm{C} \alpha$ atoms to characterize the binding cavity and to identify the most important variable regions. In particular, tautomeric, rotameric, protonation states and different 3D positions of Asp26, Leu215, Asp224, His227, Leu228, Thr274, Arg276, Arg282, Arg359 and Gly360 were carefully examined. Following the completion of the molecular alignment processes, the discodermolide conformations generated were individually inspected and no conformational problem was identified. When considering all possible intermolecular interactions of the compounds, the receptorbased flexible alignment represented the best overall structural alignment strategy, while the rigid-body fit alignment was characterized for a specific superposition region around a pre-defined common substructure, as can be observed in Figures 3 and 4. Importantly, the 3D molecular differences in the two distinct alignment approaches were quantitatively investigated using the CoMFA method, through its steric and electrostatic fields.

\section{D QSAR models}

The CoMFA method is based on the assumption that changes in ligand-binding affinities are related to changes in molecular properties represented by 3D molecular fields. Therefore, in order to investigate the structural and chemical features related to the biological activity of this series of discodermolide analogs, the two molecular alignments developed in this work were analyzed using the CoMFA steric and electrostatic fields. ${ }^{31,45}$ The structural alignments depicted in Figures 3 and 4 were employed in several PLS analyses, and the models were optimized through the CoMFA region focusing method. The region focusing was weighted by $\mathrm{StDev}^{*}$ Coefficient values ranging from 0.3 to 1.5 and grid spacing ranging from 0.5 to 2.0. This strategy not only increased $q^{2}$ values during the process of model generation, but also resulted in the refinement of $3 \mathrm{D}$ contour maps. The best statistical results are presented in Table 2 . As can be seen, significant correlation coefficients were obtained, with a cross-validated correlation coefficient $q^{2}$ of 0.68 and a conventional non-cross-validated correlation coefficient $r^{2}$ of 0.94 for the rigid-body fit alignment, while a $q^{2}$ of 0.63 and an $r^{2}$ of 0.91 for the receptor-based alignment. LMO procedures were performed in both cases as a more rigorous test for the stability and statistical significance of the models. Accordingly, the data set was divided into $10\left(\mathrm{LMO}_{10}\right)$ and $5\left(\mathrm{LMO}_{5}\right)$ randomly selected groups and, subsequently, each group was left out during 
the cross-validation process. Each model was evaluated 25 times by measuring its accuracy in predicting the activity of the remaining $10 \%$ and $20 \%$ data set compounds. The results confirmed the stability and reliability of the $3 \mathrm{D}$ QSAR models, considering that the statistical values obtained for the LMO analyses were comparable to those of the LOO analyses. Progressive scrambling of the data set was also carried out to determine the sensitivity of the QSAR models to chance correlations and to test the stability of the models. The results further confirmed the consistency of the models as defined by the critical slope, and optimum statistics for $\operatorname{cSDEP}$ and $\mathrm{Q} * * 2$ obtained at the end of different runs. In sum, the models generated in this study are very stable and possess substantial statistical significance and high internal predictive ability.

Table 2. COMFA results

\begin{tabular}{lccccccc}
\hline \multirow{2}{*}{ Alignment } & \multirow{2}{*}{$q^{2}$} & $N$ & $r^{2}$ & SEE & $r^{2}$ & \multicolumn{2}{c}{ Fraction } \\
& & & & & & $\mathrm{S}$ & $\mathrm{E}$ \\
\hline Rigid-body fit & 0.68 & 6 & 0.94 & 0.23 & 0.68 & 0.30 & 0.70 \\
Receptor-based & 0.63 & 6 & 0.91 & 0.31 & 0.85 & 0.53 & 0.47 \\
\hline
\end{tabular}

$q^{2}$, Leave-one-out (LOO) cross-validated correlation coefficient; $N$, optimum number of components; $r^{2}$, non-cross-validated correlation coefficient; SEE, standard error of estimate; $r_{\text {pred }}^{2}$, external prediction coefficient; S, steric field; E, electrostatic field.

Although the $q_{\text {LOO }}^{2}$ and $q_{\text {LMO }}^{2}$ procedures may give a suitable representation of the predictive power of the models for untested tubulin modulators, the external validation process can be considered the most valuable validation method. ${ }^{19-25,43,44}$ Therefore, the predictive ability of the internally consistent CoMFA models generated employing the 33 training set molecules (compounds 1-33, Table 1) was assessed using an external test set containing 9 compounds (compounds 33-42, Table 1), which were completely excluded from model generation. Prior to prediction, the test set compounds were processed identically to the training set compounds. On the basis of the appropriate representation of chemical diversity and distribution of property values (Table 1 and Figure 6), the test set meets the requirements for the purpose of external model validation. The detailed list of experimental and predicted $\mathrm{pIC}_{50}$ values are shown in Table 3 and the graphic results simultaneously displayed in Figure 7. Predictive $r^{2}$ values of 0.68 and 0.85 were obtained, respectively, for the rigid-body fit and receptor-based models (Table 2). Hence, in addition to good statistical quality and internal consistency, the models show high predictive power for novel $\beta$-tubulin modulators within this structural diversity. The good agreement between experimental and predicted $\mathrm{pIC}_{50}$ values also indicates the robustness of the predictive QSAR models.
Table 3. Experimental and predicted activities $\left(\mathrm{pIC}_{50}\right)$ with residual values for the test set compounds. Rigid-body fit (i) and receptor-based (ii) structural alignments

\begin{tabular}{lccccc}
\hline Comp. Experimental & $\begin{array}{c}\text { Predicted }^{2} \\
\text { CoMFA }_{(\mathrm{i})}{ }^{2}\end{array}$ & $\begin{array}{c}\text { CoMFA }_{(\mathrm{i})} \\
\text { residuals }^{\mathrm{b}}\end{array}$ & $\begin{array}{c}\text { Predicted } \\
\text { CoMFA }_{(\mathrm{ii})}\end{array}$ & $\begin{array}{c}\mathrm{CoMFA}_{(\mathrm{ii})} \\
\text { residuals }^{\mathrm{b}}\end{array}$ \\
\hline $\mathbf{3 4}$ & 5.40 & 5.93 & -0.53 & 5.90 & -0.50 \\
$\mathbf{3 5}$ & 6.29 & 6.86 & -0.57 & 6.17 & 0.12 \\
$\mathbf{3 6}$ & 7.00 & 6.73 & 0.27 & 7.01 & -0.01 \\
$\mathbf{3 7}$ & 7.89 & 6.80 & 1.09 & 7.19 & 0.70 \\
$\mathbf{3 8}$ & 5.70 & 6.02 & -0.32 & 5.83 & -0.13 \\
$\mathbf{3 9}$ & 8.10 & 7.69 & 0.41 & 7.94 & 0.16 \\
$\mathbf{4 0}$ & 6.51 & 6.52 & -0.01 & 5.97 & 0.54 \\
$\mathbf{4 1}$ & 7.24 & 7.44 & -0.20 & 7.22 & 0.02 \\
$\mathbf{4 2}$ & 6.80 & 7.14 & -0.34 & 6.76 & 0.04 \\
\hline
\end{tabular}

${ }^{\mathrm{a}} \mathrm{pIC}_{50}$ values. ${ }^{\mathrm{b}}$ The difference between experimental and predicted values.
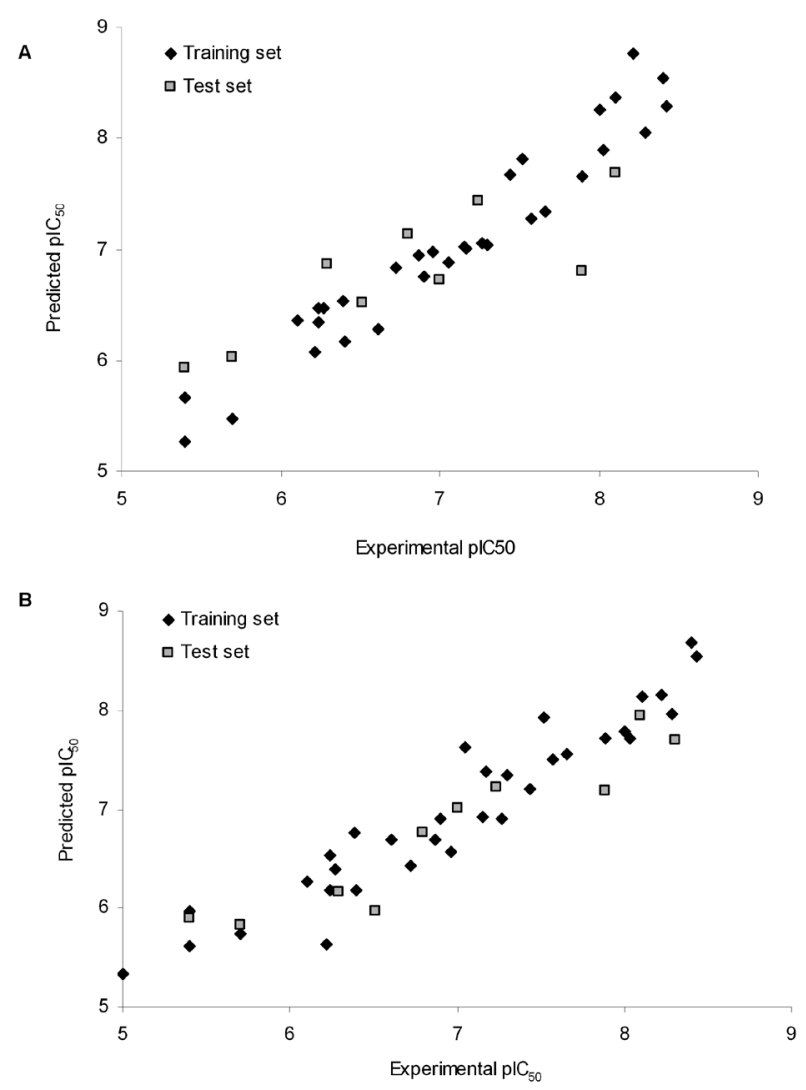

Figure 7. Plot of predicted values of $\mathrm{pIC}_{50}$ versus the corresponding experimental values for the training (black squares) and test (gray squares) compounds for rigid-body fit (A) and receptor-based (B) structural alignments.

As can be seen, the receptor-based CoMFA model possesses higher predictive ability than the corresponding rigid-body fit model, as indicated by its predictive $r^{2}$ value and lower residual values (Table 3 ). In addition, the relative contributions of the steric and electrostatic fields 
to the biological activity are different for the two models. According to the data of Table 2, the CoMFA steric field descriptor for the rigid-body fit model explained $30 \%$ of the variance, while the electrostatic descriptor accounted for the remaining $70 \%$. For the receptor-based model, the corresponding steric and electrostatic fields explained $53 \%$ and $47 \%$, respectively. The results of the receptor-based CoMFA model are in accordance with previous classical and 3D QSAR studies on the interaction of taxanes in the tubulin/microtubule system, which indicated that the hydrophobic feature of the substituents along with the steric parameters/fields are the most important determinants of biological activity and directly reflect the chemical environment of the tubulin binding pocket. ${ }^{27,28}$

In addition to prediction of property values for the test set molecules, the derived 3D QSAR CoMFA models should also provide insights into molecular properties intrinsically related to receptor-binding affinity and biological activity. One prominent feature of the CoMFA method is the easy visualization of the steric and electrostatic regions in space responsible for increases and decreases in the values of a particular type of dependent variable (e.g., $\mathrm{pIC}_{50}$ ). The 3D contour maps generated in our studies represent an important tool for the understanding of specific tubulin-discodermolide interactions related to the antimitotic activity.

The specialized molecular fields can be analyzed considering two aspects in the CoMFA contour maps. Unfavorable steric regions are represented in yellow and favorable steric regions in green, while red contours represent regions where electronegative substituents may increase the biological activity, and blue contours indicate regions where electropositive groups would contribute to enhance the antimitotic potency. For comparison purposes, the CoMFA electrostatic contour maps for the rigid-body fit and receptor-based models are shown in Figure 8 as PLS StDev*Coefficient plots, using the discodermolide analog 32 (Table 1). Although the importance of the $\mathrm{C}_{19}$-carbamate and lactone carbonyl groups, as well as the electronegative substituents near $\mathrm{C}_{17}$ is emphasized in both alignment models, only the receptor-based model present a red contour surrounding the $(S)-\mathrm{C}_{7}$ region, indicating that electronegative groups (hydrogen bond acceptors) are related to increasing antimitotic potency. These observations are in agreement with prior experimental evidence that suggests the importance of the substituents at $\mathrm{C}_{19}$-carbamate, $(R)-\mathrm{C}_{17}$-hydroxyl, and $(S)-\mathrm{C}_{7}$-hydroxyl, and the carbonyl oxygen of the lactone ring to the observed discodermolide cytotoxicity. ${ }^{25,37,46,47}$

The corresponding steric fields for the two generated molecular alignments are depicted in Figure 9. The green contours surrounding the lactone ring of the discodermolide
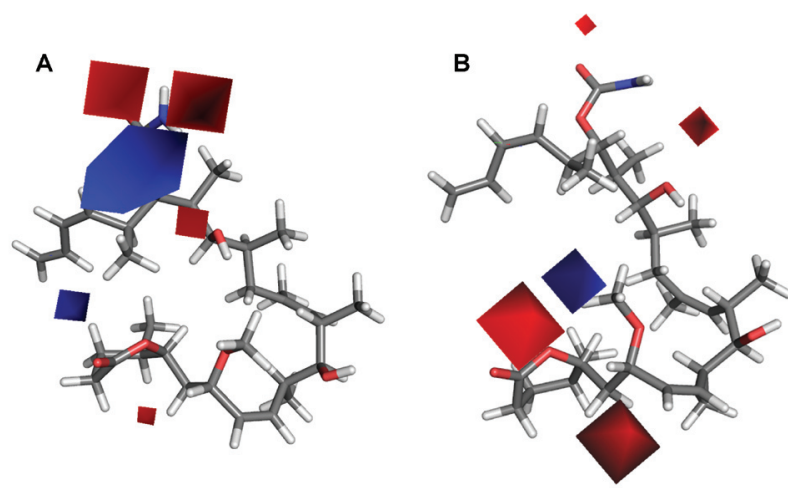

Figure 8. CoMFA electrostatic contour maps (as SD $\times$ coefficient). (A) Rigid-body fit alignment. (B) Receptor-based alignment. Higher values of $\mathrm{pIC}_{50}$ are correlated to more positive charge near blue and more negative charge near red. Compound $\mathbf{3 2}$ is displayed in the background for reference.

analog (compound 32, Table 1) suggest that bulky substituents would be favorable in that region according to both rigid-body fit and receptor-based steric contour maps. However, the receptor-based contour map explained this steric region in more detail, revealing that bulkier groups surrounding the lactone ring region would be detrimental for antimitotic potency, as emphasized by the unfavorable steric regions depicted in the yellow maps. Furthermore, the $\mathrm{C}_{14}$-substituent was found to be important to increasing cytotoxicity, and this result is also in accordance with previous studies..$^{25,48}$
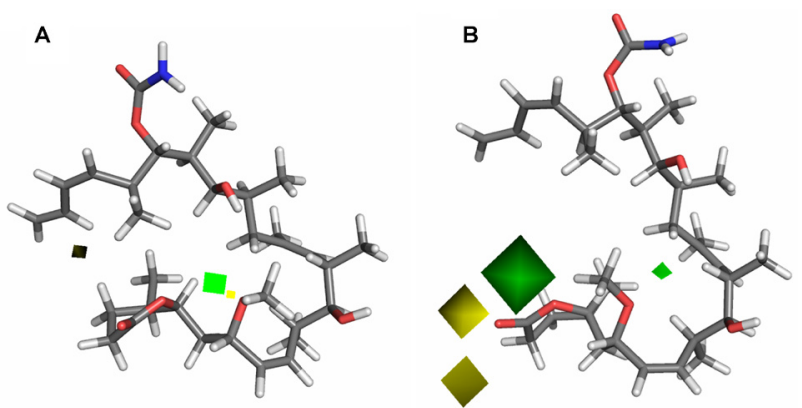

Figure 9. CoMFA steric contour maps (as SD $\times$ coefficient). (A) Rigidbody fit alignment. (B) Receptor-based alignment. Higher values of $\mathrm{pIC}_{50}$ are correlated to more bulky groups near green and less bulky groups near yellow. Compound $\mathbf{3 2}$ is displayed in the background for reference.

The $3 \mathrm{D}$ receptor-based model generated in this study is compatible with the chemical environment of the $\beta$-tubulin binding cavity, as shown in Figure 10. The importance of the $\mathrm{C}_{19}$-carbamate, $(R)-\mathrm{C}_{17}$-hydroxyl, $\mathrm{C}_{14}$-methyl and $(S)$ $\mathrm{C}_{7}$-hydroxyl substituents, and the lactone ring corroborates with the $\beta$-tubulin interaction sites Asp26, Gly360, Phe270, Arg276 and His227, as previously reported. ${ }^{25,37,46-48}$ As can be seen in the docking position obtained for discodermolide (Figure 10), the lactone ring fits in the Asp224/His227 pocket and leads to the formation of a hydrogen bond between the 
carbonyl oxygen and the side chain of Arg276, while the $\mathrm{C}_{19}$-carbamate $\mathrm{NH}_{2}$ group is oriented toward the side chain of Asp26 to form an additional hydrogen bond. The $(S)-\mathrm{C}_{7}-$ hydroxyl substituent is also hydrogen-bonded to $\operatorname{Arg} 276$ and the $\mathrm{C}_{14}$-methyl group interacts with Phe270 through hydrophobic contacts. The identification of these important structural features should be useful for the design of new structurally related MSAAs with improved potency.

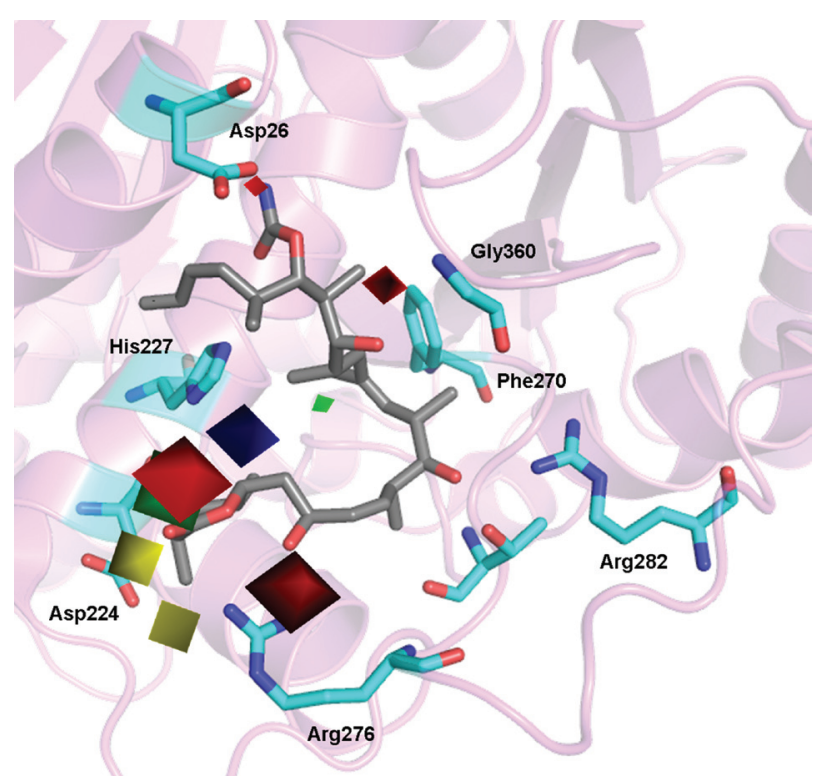

Figure 10. Three-dimensional model of discodermolide (compound 25) in the $\beta$-tubulin binding cavity along with CoMFA steric and electrostatic fields.

\section{Conclusions}

The understanding of protein-ligand intermolecular interactions is essential for the design of compounds with improved affinity and biological potency. ${ }^{17,22}$ This assumption is particularly evident in the case of the highly flexible discodermolide modulators analyzed in this study. Robust 3D QSAR CoMFA models were developed employing two distinct alignment strategies, and the models possess high internal and external consistency, showing substantial predictive power. The CoMFA contour maps emphasized important regions in 3D space where modifications of steric and electrostatic fields would be strongly associated with concomitant changes in the observed antimitotic potency. In addition, the receptorbased model generated was compatible with the $3 \mathrm{D}$ protein environment in the $\beta$-tubulin binding site and, therefore, could be used to guide further structural modifications as well as structure-based design of new $\beta$-tubulin modulators with improved affinity and potency. The integration of molecular modeling and 3D QSAR studies was a useful tool for the identification of chemical and structural features responsible for binding affinity, microtubule stabilization and antitumoral properties of this series of discodermolide derivatives.

\section{Acknowledgments}

We gratefully acknowledge financial support from the National Council for Scientific and Technological Development (CNPq, Conselho Nacional Desenvolvimento Científico e Technológico) and the State of São Paulo Research Foundation (FAPESP, Fundação de Amparo à Pesquisa do Estado de São Paulo), Brazil.

\section{References}

1. Wittmann, T.; Hyman, A.; Desai, A.; Nat. Cell. Biol. 2001, 3, E28.

2. Jordan, M. A.; Wilson, L.; Nat. Ver. Cancer 2004, 4, 253.

3. Hadfield, J. A.; Ducki, S.; Hirst, N.; McGown, A. T.; Prog. Cell Cycle Res. 2003, 5, 309.

4. Pellegrini, F.; Budman, D. R.; Cancer Invest. 2005, 23, 264.

5. Islam, M. N.; Iskander, M. N.; Mini Rev. Med. Chem. 2004, 4, 1077.

6. Snyder J. P.; Nat. Chem. Biol. 2007, 3, 81.

7. Schiff, P.B.; Fant, J.; Horwitz, S. B.; Nature 1979, $277,665$.

8. Rowinsky, E. K.; Annu. Rev. Med. 1997, 48, 353.

9. Jordan, M. A.; Toso, R. J.; Thrower, D.; Wilson, L.; Proc. Natl. Acad. Sci. U. S. A. 1993, 90, 9552.

10. Orr, G. A.; Verdier-Pinard, P.; McDaid, H.; Horwitz, S. B.; Oncogene 2003, 22, 7280.

11. He, L.; Orr, G. A.; Horwitz, S. B.; Drug Discov. Today 2001, $6,1153$.

12. Bollag, D. M.; McQueney, P. A.; Zhu, J.; Hensens, O.; Koupal, L.; Liesch, J.; Goetz, M.; Lazarides, E.; Woods, C. M.; Cancer Res. 1995, 55, 2325.

13. Hung, D. T.; Chen, J.; Schreiber, S. L.; Chem. Biol. 1996, 3, 287.

14. ter Haar, E.; Kowalski, R. J.; Hamel, E.; Lin, C. M.; Longley, R. E.; Gunasekera, S. P.; Rosenkranz, H. S.; Day, B. W.; Biochemistry 1996, 35, 243.

15. Madiraju, C.; Edler, M. C.; Hamel, E.; Raccor, B.S.; Balachandran, R.; Zhu, G.; Giuliano, K. A.; Vogt, A.; Shin, Y.; Fournier, J. H.; Fukui, Y.; Brückner, A. M.; Curran, D. P.; Day, B. W.; Biochemistry 2005, 44, 15053; Dias, L. C.; Lima, D. J. P.; Gonçalves, C. C. S.; Andricopulo, A. D.; Eur. J. Org. Chem. 2009, 1491.

16. Mínguez, J. M.; Kim, S. Y.; Giuliano, K. A.; Balachandran, R.; Madiraju, C.; Day, B. W.; Curran, D. P.; Bioorg. Med. Chem. 2003, 11, 3335.

17. Guido, R. V. C.; Oliva, G.; Andricopulo, A. D.; Curr. Med. Chem. 2008, 15, 37. 
18. Andricopulo, A. D.; Montanari, C. A.; Mini-Rev. Med. Chem. 2005, 5, 585 .

19. Salum, L. B.; Polikarpov, I.; Andricopulo, A. D.; J. Mol. Graphics Modell. 2007, 26, 43.

20. Castilho, M. S.; Postigo, M. P.; de Paula, C. B.; Montanari, C. A.; Oliva, G.; Andricopulo, A. D.; Bioorg. Med. Chem. 2006, $14,516$.

21. Andrade, C. H.; Salum, L. B.; Pasqualoto, K. F. M.; Ferreira, E. I.; Andricopulo, A. D.; Lett. Drug Des. Discov. 2008, 5, 377.

22. Guido, R. V. C.; Oliva, G.; Montanari, C. A.; Andricopulo, A. D.; J. Chem. Inf. Model. 2008, 48, 918.

23. Salum, L. B.; Polikarpov, I.; Andricopulo, A. D.; J. Chem. Inf. Model. 2009, 48, 2243.

24. Honório, K. M.; Garratt, R. C.; Polikarpov, I.; Andricopulo, A. D.; J. Mol. Graph. Modell. 2007, 25, 921.

25. Salum, L. B.; Dias, L. C.; Andricopulo, A. D.; QSAR Comb. Sci. 2009, 28, 325.

26. Castilho, M. S.; Guido, R. V. C.; Andricopulo, A. D.; Lett. Drug Des. Discov. 2007, 4, 106.

27. Manetti, F.; Maccari, L.; Corelli, F.; Botta, M.; J. Mol. Model. 2005, 11, 48.

28. Hansch, C.; Verma, R. P.; Mol. Pharm. 2008, 5, 151.

29. Manetti, F.; Maccari, L.; Corelli, F.; Botta, M.; Curr. Top. Med. Chem. 2004, 4, 203.

30. Cunningham, S. L.; Cunningham, A. R.; Day, B. W.; J. Mol. Model. 2005, 11, 48.

31. Cramer, R. D.; Patterson, D. E.; Bunce, J. D.; J. Am. Chem. Soc. 1988, 110, 5959.

32. Burlingame, M. A.; Shaw, S. J.; Sundermann, K. F.; Zhang, D.; Petryka, J.; Mendoza, E.; Liu, F.; Myles, D. C.; LaMarche, M. J.; Hirose, T.; Scott Freeze, B.; Smith, A. B. III.; Bioorg. Med. Chem. Lett. 2004, 14, 2335.

33. Shaw, S. J.; Sundermann, K. F.; Burlingame, M. A.; Myles, D. C.; Freeze, B. S.; Xian, M.; Brouard, I.; Smith, A. B. III.; J. Am. Chem. Soc. 2005, 127, 6532.

34. Smith, A. B. III; Freeze, B. S.; Lamarche, M. J.; Hirose, T.; Brouard, I.; Rucker, P. V.; Xian, M.; Sundermann, K. F.; Shaw, S. J.; Burlingame, M. A.; Horwitz, S. B.; Myles, D. C.; Org. Lett. 2005, 7, 311.
35. Smith, A. B. III; Freeze, B. S.; Lamarche, M. J.; Hirose, T.; Brouard, I.; Xian, M.; Sundermann, K. F.; Shaw, S. J.; Burlingame, M. A.; Horwitz, S. B.; Myles, D. C.; Org. Lett. 2005, 7, 315 .

36. Smith, A. B. III; Xian, M.; Org. Lett. 2005, 7, 5229.

37. Martello, L. A.; LaMarche, M. J.; He, L.; Beauchamp, T. J.; Smith, A. B.; Horwitz, S. B.; Chem. Biol. 2001, 8, 843.

38. Gasteiger, J.; Marsili, M.; Tetrahedron 1980, 36, 3219.

39. Clark, M.; Cramer, R. D.; Van Opdenbosch, N.; J. Comput. Chem. 1989, 10, 982.

40. Verdonk, M.L.; Cole, J.C.; Hartshorn, M.J.; Murray, C.W.; Taylor, R.D.; Proteins 2003, 52, 609.

41. Snyder, J. P.; Nettles, J. H.; Cornett, B.; Downing, K. H.; Nogales, E.; Proc. Natl. Acad. Sci. U. S. A. 2001, 98, 5312

42. Nettles, J. H.; Li, H.; Cornett, B.; Krahn, J. M.; Snyder, J. P.; Downing, K. H.; Science 2004, 305, 866.

43. Golbraikh, A.; Shen, M.; Xiao, Z.; Xiao, Y. D.; Lee, K. H.; Tropsha, A.; J. Comput. -Aided Mol. Des. 2003, 17, 241.

44. Golbraikh, A.; Tropsha, A.; J. Mol. Graphics Modell. 2002, 20 , 269.

45. Cramer, R. D.; Patterson, D. E.; Bunce, J. D.; Prog. Clin. Biol. Res. 1989, 291, 161.

46. Xia, S.; Kenesky, C. S.; Rucker, P. V.; Smith, A. B. III; Orr, G. A.; Horwitz, S. B.; Biochemistry 2006, 45, 11762.

47. Smith, A. B. III; LaMarche, M. J.; Falcone-Hindley, M.; Org. Lett. 2001, 3, 695.

48. Shin, Y.; Fournier, J. H.; Balachandran, R.; Madiraju, C.; Raccor, B. S.; Zhu, G.; Edler, M. C.; Hamel, E.; Day, B. W.; Curran, D. P.; Org. Lett. 2005, 7, 2873.

Received: November 5, 2008 Web Release Date: April 24, 2009

FAPESP helped in meeting the publication costs of this article. 